\title{
Energy-Filtering Transmission Electron Microscopy for the Characterization of Polymer Blend Morphologies
}

\author{
Shin HoriuchI ${ }^{\dagger}$ Kiyoshi Yase, Takeshi Kitano, \\ Noboru Higashida, ${ }^{*}$ and Toshiaki OugizaWA* \\ National Institute of Materials and Chemical Research, \\ 1-1 Higashi, Tsukuba, Ibaraki 305, Japan \\ * Department of Organic and Polymeric Materials, Tokyo Institute of Technology, \\ Ookayama, Meguro-ku, Tokyo 152, Japan
}

(Received August 22, 1996)

\begin{abstract}
KEY WORDS Energy-Filtering Transmission Electron Microscopy (EFTEM) / Electron Energy Loss Spectroscopy (EELS) / Electron Spectroscopic Imaging (ESI) / Polymer Blend / Morphology /
\end{abstract}

Much attention has been recently focused on energyfiltering transmission electron microscopy (EFTEM) as an analytical microscopy having a spatial resolution of less than a nanometer. ${ }^{-3}$ The EFTEM allows us to analyze element compositions by electron energy loss spectroscopy (EELS) and allows us to create an image by electron spectroscopic imaging (ESI) mode. The EELS gives us information on both qualitative and quantitative elemental compositions at local area of materials from the edge profiles of element specific ionization of inner shells. The ESI is an imaging technique which creates images with the unscattered electrons or the inelastically scattered electrons at selective energy losses. The "contrast tuning" and the "element specific contrast" obtained by ESI are a part of the useful features of ESI. 4 There are several works regarding the application of EFTEM to the morphological studies of multiphase polymer materials. Cantow et al. firstly attempted the imaging of distributions of low atomic number elements in some multiphase polymer materials with ESI mode in EFTEM. ${ }^{5-7}$ However, no works have been appeared regarding the elemental distribution imaging for the polymers which have been frequently used as components for polymer alloys and blends such as polyamide (PA) and poly(methylmethacrylate) (PMMA). The reason for that may be the low sensitivity of $\mathrm{N}$ and $\mathrm{O}$ in EELS in specimens where carbon is the predominant element, ${ }^{8}$ the loss of light elements through the radiation damage ${ }^{9}$ and the difficulties for the preparation of thin unstained specimens especially for PA to be necessary for the reduction of the plural inelastic scattering. ${ }^{10}$ Preparing thin unstained specimens less than $50 \mathrm{~nm}$ and introducing a "Imaging Plate" (IP) system for a recording medium, we here present the imaging of morphologies by elemental mapping in the blends of polyamide6 (PA6)/polycarbonate (PC) and polystyrene (PS)/PMMA. The IP has been recently developed as a recording medium for TEM. This system enables us to record a image at room temperature with very little irradiation damage by reducing electron dose to a specimen. ${ }^{11}$ Moreover, we evaluate the quantitative aspects of EELS using the IP system. The IP allows us to record a electron energy loss spec-

† To whom correspondence should be addressed. trum (EEL-spectrum) with wide range of energy losses more than $100 \mathrm{eV}$, with faster acquisition time less than one second and with wide dynamic range covering the electron dose from $2 \times 10^{-14}$ to $2 \times 10^{-9} \mathrm{Ccm}^{-2} .^{12}$ Hence physical drift and radiation damage of specimens which have been always occurred in EELS recording can be minimized.

\section{EXPERIMENTAL}

The samples used in this study are the blends of polyamide6 and polycarbonate (PA6/PC) and polystyrene/ poly(methylmethacrylate) (PS/PMMA). The blends of PA6/PC (75/25 in weight ratio) and PS/PMMA (85/15) were prepared by melt mixing for $10 \mathrm{~min}$ at $260^{\circ} \mathrm{C}$ and at $200^{\circ} \mathrm{C}$, respectively, using a miniature mixer.

The specimens for EFTEM observation were prepared by cryo-microtoming at $-110^{\circ} \mathrm{C}$ using a diamond knife. The specimens with a thickness of $50 \mathrm{~nm}$ or less were used for the observation.

EFTEM used in this study was a Zeiss CEM 902 instrument equipped with a Castaing-Henry electronenergy filter, using an acceleration voltage of $80 \mathrm{kV}$. The basic construction has been described in detail elsewhere. ${ }^{13,14}$ The observation was carried out at room temperature. An IP System, FDL5000, Fuji Photofilm Co., Ltd., was introduced for recording of energy-filtering images and EEL-spectra. To avoid the significant radiation damage of specimens, the exposure level including set-up time was kept less than $10^{-2} \mathrm{C} \mathrm{cm}^{-2}$. The area of interest in an image is determined by an area-selecting aperture just above the energy-filter and then the spectra were recorded on an IP. The profiles of the spectra were then obtained by IP-processor in this system.

\section{RESULTS AND DISCUSSION}

Figure 1a shows a zero-loss filtered image (zero-loss image) of an unstained specimen of PA6/PC 75/25 blend. The energy-selecting slit is centered at the zero-loss peak of energy width of $10 \mathrm{eV}$ formed by the unscattered and elastically scattered electrons. Although the domains and the matrix can be identified by the voids at the boundary 

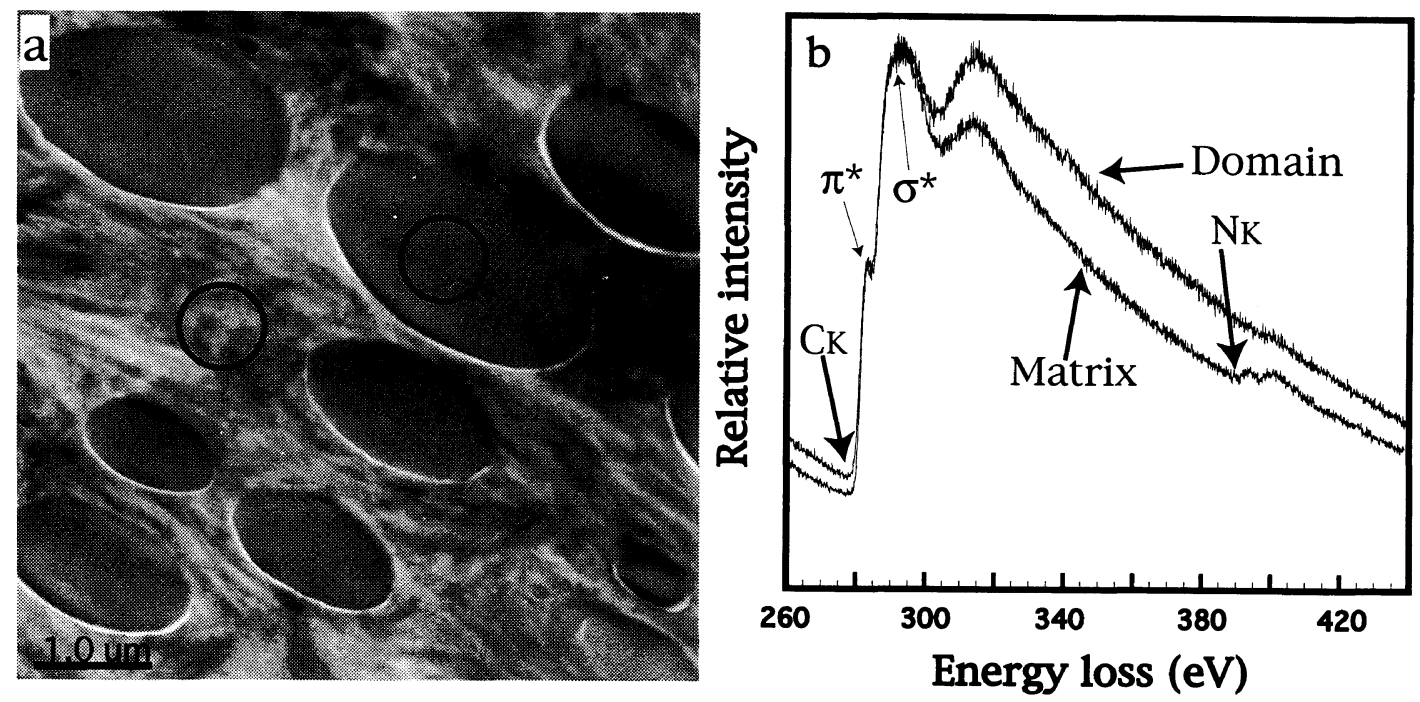

Figure 1. (a) A zero-loss image showing the morphology of the PA6/PC 75/25 blend with an unstained specimen and (b) EEL-spectra showing carbon $\mathrm{K}$-edge and nitrogen $\mathrm{K}$-edge obtained from the regions indicated as circles in Figure 1a.

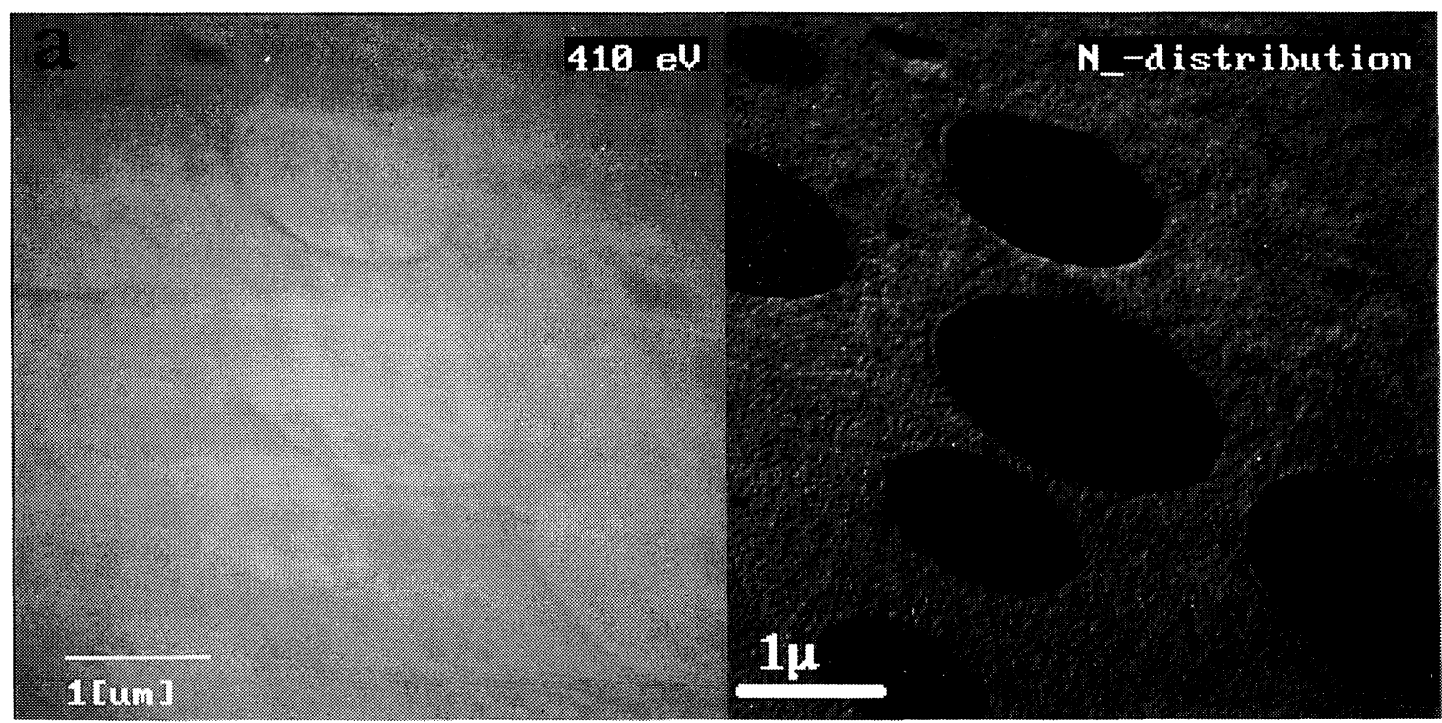

Figure 2. (a) A energy-filtering image of the PA6/PC $75 / 25$ blend formed by electrons with an energy-loss of $410 \pm 5 \mathrm{eV}$ and (b) a nitrogen distribution image obtained by the three window power law method.

between the domains and the matrix, the contrast of this image is low. The spectra shown in Figure $1 \mathrm{~b}$ show carbon $\mathrm{K}$-edge taken from the regions indicated as circles in Figure 1a. These spectra were recorded on IP with an electron dose to the specimen of 110 electrons $\mathrm{nm}^{-2}$. The profile of the carbon $\mathrm{K}$-edge involves both the $\pi^{*}$ state $(285 \mathrm{eV})$ and the $\sigma^{*}$ state $(290 \mathrm{eV})$. The spectrum from the matrix shows the nitrogen $\mathrm{K}$-edge at around $400 \mathrm{eV}$, which means that PA6 forms matrix and that PA6 and $\mathrm{PC}$ are clearly segregated from each other. Figure 2a shows a energy-filtering image formed by electrons with an energy-loss of $410 \pm 5 \mathrm{eV}$ (nitrogen core-loss image). Due to the low concentration of nitrogen in PA6, the intensity of the peak near the nitrogen K-edge cannot surpass the background intensity of PC phase as shown in Figure $1 \mathrm{~b}$ and hence the contrast of this image is quite low. To obtain the nitrogen distribution image, the background intensity has to be subtracted from the nitrogen core loss image. In order for that, we introduced the "three window power law method."15
In this method the background intensity $S(E)$ is assumed to have an inverse power-law dependence on energy loss $E$ as follows:

$$
S(E)=A E^{-r} .
$$

The constant factors $A$ and $r$ can be calculated pixel by pixel from the signals at two energy windows $E_{\mathrm{w} 1}$ and $E_{\mathrm{w} 2}$ below the edge. Then the background is extrapolated to the edge. The net signal $S_{\mathrm{N}}$ is thus given by

$$
S_{\mathrm{N}}=S\left(E_{\max }\right)-A E_{\max }^{-r}
$$

where $E_{\max }$ is the energy window beyond the edge giving the signal $S\left(E_{\max }\right)$. For the imaging of nitrogen distribution in the blend of PA6/PC, energy-filtering images formed by electrons with an energy-loss of $340 \pm 5 \mathrm{eV}$ and $392 \pm 5 \mathrm{eV}$ are recorded to create background signals and then the nitrogen distribution image is successfully obtained as shown in Figure $2 \mathrm{~b}$. This image reveals that the PA6 phase can be visualized as the nitrogen distribution image from an unstained specimen. 
Introducing the same procedure, we obtained an oxygen distribution image in the blend of PS/PMMA. Figure 3 shows the EEL-spectra in the region of carbon $\mathrm{K}$-edge taken from the PS matrix and from a PMMA domain in the PS/PMMA $85 / 15$ blend. The difference of the intensities of the spectra beyond the carbon $\mathrm{K}$-edge reflects the difference of the carbon concentration in the two polymers. As shown also in Figure 4, oxygen K-edge can be detected only in the spectrum from the PMMA domains. Hence, the oxygen distribution image for the PS/PMMA blend should be corresponded to the PMMA distribution image. Using the energy-filtering images formed by electrons with an energy-losses of $480 \pm 5 \mathrm{eV}$ and $526 \pm 5 \mathrm{eV}$ for the background, the oxygen distribution image can be obtained from the oxygen coreloss image at $544 \pm 5 \mathrm{eV}$ (Figure $4 \mathrm{a}$ ) as shown in Figure $4 \mathrm{~b}$. Introducing the "three window power law method" in ESI, we successfully obtained elemental mapping images from the blends of PA6/PC and PS/PMMA. This suggests that EFTEM offers an alternative technique for heavy metal staining to visualize polymer phases.

Quantitative aspects of EEL-spectra taken from the specimens mentioned above were evaluated. The ratio of the number of elements was quantified using the following expression ${ }^{16}$ :

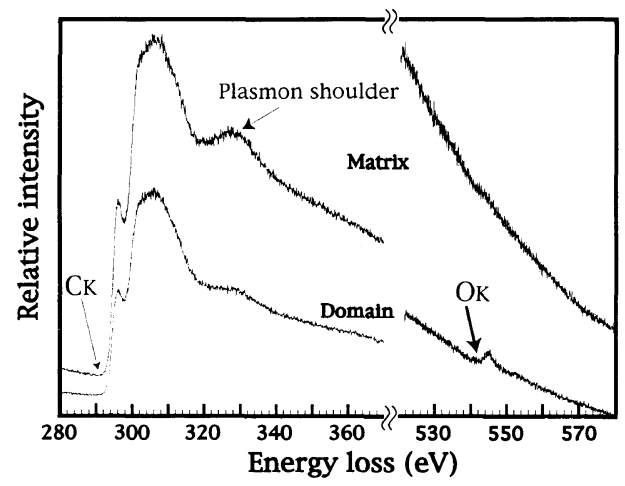

Figure 3. EEL-spectra from a domain and from the matrix in the blend of $85 / 15$ PS/PMMA.

$$
\frac{N_{1}}{N_{2}}=\frac{I_{1}(\alpha, \Delta)}{I_{2}(\alpha, \Delta)} \frac{\sigma_{2}(\alpha, \Delta)}{\sigma_{1}(\alpha, \Delta)}
$$

where $N_{\mathrm{i}}$ is the absolute number of detected atoms, the superscripts 1 and 2 represent the elemental species, $\sigma$ is the atomic ionization cross section, $\alpha$ is the collection angle which depends on the size of an objective aperture, $\Delta$ is the integration energy window and $I$ is the measured intensity. The integrated intensity was obtained by extrapolating the pre-edge background by means of the inverse power law. In this study, the integration energy window, $\Delta$, was set at $30 \mathrm{eV}$ and the ionization cross sections, $\sigma$, for carbon, nitrogen and oxygen are assumed to be comparable with regard to the report by Wall et $a l .{ }^{17}$ Hence, the ratio of the integrated intensities of the edges should be equivalent to the atomic ratio of elements per unit area. Table I shows the results of the quantification and the comparison with the calculated values. These results are the average of at least five positions in the specimens. For the two blend systems, the atomic ratios of carbon in the respective phases show good agreement to the calculated values, while the ratio of nitrogen to carbon in PA6 phase and that of oxygen to carbon in PMMA phase are much smaller than the calculated values. Although the EEL-spectra were taken at low electron dose, the mass loss of the specimens by radiation damage cannot be avoided and hence the detected nitrogen and oxygen core-loss peaks are weak. And also, the process for the extrapolation of the preedge background for the nitrogen and oxygen edges is

Table I. Results of quantification of EEL-spectra and the comparison with calculated values

\begin{tabular}{cccccc}
\hline Sample & $\begin{array}{c}\text { Atomic ratio of carbon } \\
\text { in respective phases } \\
\text { in the blends }\end{array}$ & & \multicolumn{2}{c}{$\begin{array}{c}\text { Atomic ratio of nitrogen to } \\
\text { carbon in PA6 or oxygen } \\
\text { to carbon in PMMA }\end{array}$} \\
\cline { 2 - 3 } \cline { 5 - 6 } & Exptl & Calcd & & Exptl & Calcd \\
\hline PA6/PC & 0.92 & 0.88 & & 0.050 & 0.17 \\
PS/PMMA & 1.71 & 1.76 & & 0.040 & 0.40 \\
\hline
\end{tabular}

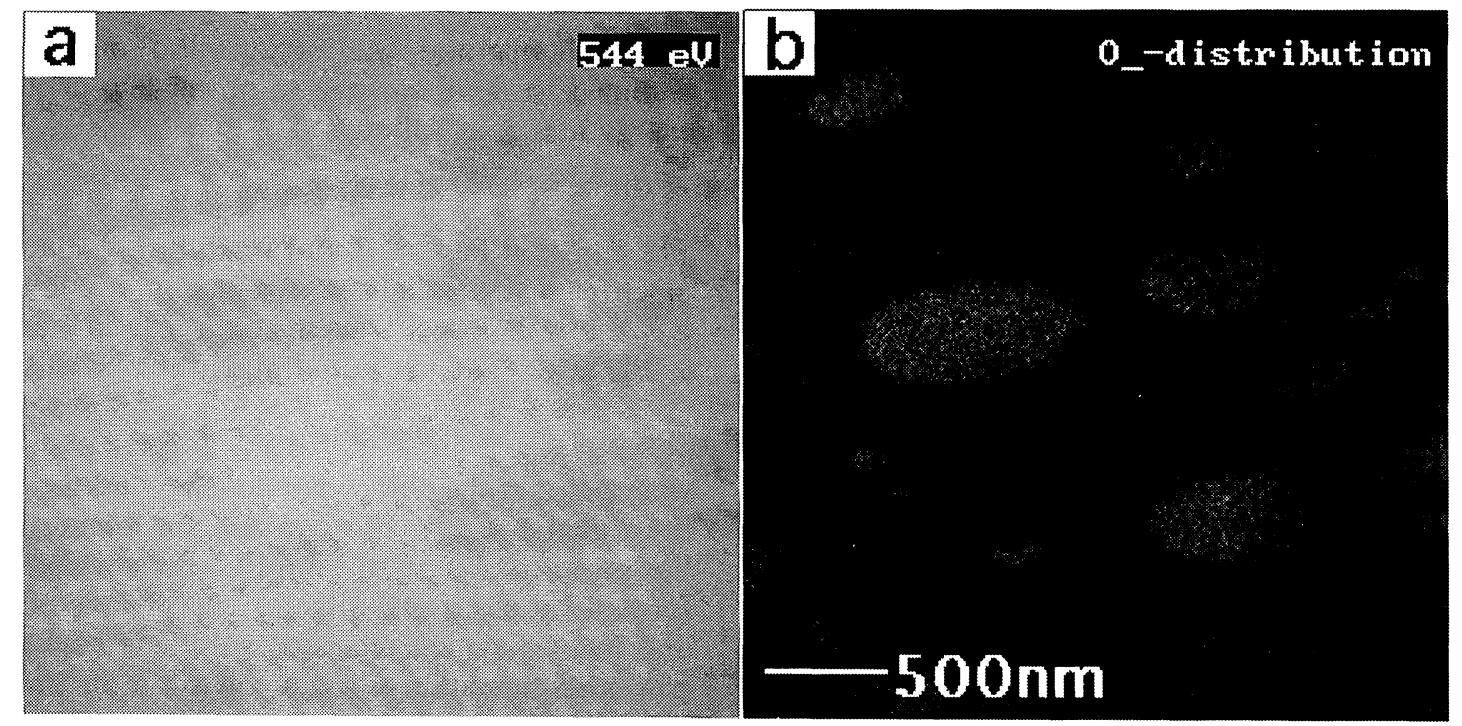

Figure 4. (a) A energy-filtering image of the PS/PMMA $85 / 15$ blend formed by electrons with an energy-loss of $544 \pm 5$ eV. (b) A oxygen distribution image obtained by three window power law method. 
the another reason for this disagreement. The carbon $\mathrm{K}$-edge is a dominant feature of the EEL spectra in a specimen where carbon is the predominant element. The background of nitrogen and oxygen edges contains the tail of preceding carbon ionization edge. The plasmon shoulder appeared beyond the carbon $\mathrm{K}$-edge, indicated in Figure 3, due to electrons which suffered an additional energy loss from outer shell excitations increases with the specimen thickness. ${ }^{10}$ This alters the shape of the carbon peak and affects to the background of nitrogen and oxygen $\mathrm{K}$-edges. In this case simply fitting the background to a power law is not feasible. We insist here that the quantification of carbon, which contains as the predominant element, will be useful to characterize the phase features when the concentration of carbon in each component is different. In order to quantify minor elements, however, specimens have to be thin enough to reduce plural inelastic scattering and should be cooled to reduce the radiation damage.

\section{REFERENCES}

1. L. M. Brown, Nature, 366, 721 (1993).

2. D. A. Muller, Y. Tzou, R. Raj, and J. Silcox, Nature, 366, 725 (1993).
3. P. E. Batson, Nature, 366, 727 (1993).

4. S. Horiuchi, N. Matchariyakul, K. Yase, T. Kitano, H. K. Choi, and Y. M. Lee, Polymer, 37, 3065 (1996).

5. M. Kunz, M. Moller, and H. J. Cantow, Makromol. Chem., Rapid Commun., 8, 401 (1987).

6. M. Kunz, M. Moller, U. R. Heinrich, and H. J. Cantow, Makronol. Chem., Macromol. Symp., 23, 57 (1989).

7. H. J. Cantow, M. Kunz, S. Klotz, and M. Moller, Makronol. Chem., Macromol. Symp., 26, 191 (1989).

8. N. Shiraga, K. Tanaka, and M. Minobe, Kobunshi Ronbunshu, 49, 353 (1992).

9. L. C. Sawyer and D. T. Grubb, "Polymer Microscopy," Chapmann and Hall, London, 1987, p 62.

10. R. Door, K. D. Haberle, and R. Martin, J. Microscopy, 174, 183 (1994)

11. H. Ayato, N. Mori, J. Miyahara, and T. Oikawa, J. Electron Microscopy, 39, 444 (1990).

12. N. Mori, T. Oikawa, Y. Harada, and J. Miyahara, J. Electron Microscopy, 39, 433 (1990).

13. H. Rose and D. Krahl, "Energy-Filtering Transmission Electron Microscopy," L. Reimer, Ed., Springer, Heidelberg, 1995, p 43.

14. R. Bauer, "Method in Microbiology," F. Mayer, Ed., Academic Press, London, 1988, p 113.

15. L. Reimer, "Energy-Filtering Transmission Electron Microscopy," L. Reimer, Ed., Springer, Heidelberg, 1995, p 347.

16. F. Egerton and R. D. Leapman, "Energy-Filtering Transmission Electron Microscopy," L. Reimer, Ed., Springer, Heidelberg, 1995, p 269.

17. L. Reimer, "Energy-Filtering Transmission Electron Microscopy," L. Reimer, Ed., Springer, Heidelberg, 1995, p 12. 